\title{
Pediatric extracorporeal membrane oxygenation (ECMO): a guide for radiologists
}

\author{
Adrienne F. Thompson ${ }^{1} \cdot$ Jiali Luan ${ }^{2} \cdot$ Mohammed M. Al Aklabi ${ }^{3} \cdot$ Dominic A. Cave $^{4} \cdot$ Lindsay M. Ryerson $^{5}$. \\ Michelle L. Noga ${ }^{1}$
}

Received: 12 January 2018 / Revised: 16 April 2018 / Accepted: 12 July 2018 / Published online: 6 August 2018

(C) Springer-Verlag GmbH Germany, part of Springer Nature 2018

\begin{abstract}
Extracorporeal membrane oxygenation (ECMO) is a life-saving treatment for pediatric patients with respiratory and/or cardiac failure. The ECMO circuit oxygenates and sometimes pumps the blood, effectively replacing lung and/or heart function temporarily. ECMO patients are clinically very complex not only because of their underlying, life-threatening pathology, but also because of the many physiological parameters that must be monitored and adjusted to maintain adequate tissue perfusion and oxygenation. Drainage and reinfusion cannulae connecting the patient to the ECMO circuit are visible on radiograph. These cannulae have different functions, different configurations, different radiographic appearances, and different positions that should be familiar to the interpreting pediatric radiologist. The primary complications of ECMO include hemorrhage, thrombosis and ischemia, as well as equipment failure and cannula malpositioning, all of which may be detected on imaging. In this pictorial essay, we discuss the basics of ECMO function and clinical management, ECMO cannula features and configurations, and the many complications of ECMO from an imaging perspective. Our goal is to educate pediatric radiologists about ECMO imaging, equipping them to properly interpret these studies and to become a useful consultant in ECMO patient care.
\end{abstract}

Keywords Cardiopulmonary bypass $\cdot$ Children $\cdot$ Extracorporeal membrane oxygenation $\cdot$ Infant $\cdot$ Life support

\section{Introduction}

Extracorporeal membrane oxygenation (ECMO) is a form of extracorporeal life support (ECLS) that provides cardiopulmonary bypass. It oxygenates and sometimes pumps the blood in the setting of decompensated respiratory and/or cardiac failure. The first successful use of prolonged ECMO was in a young

CME activity This article has been selected as the CME activity for the current month. Please visit the SPR website at www.pedrad.org on the Education page and follow the instructions to complete this CME activity.

Adrienne F. Thompson

thompson@ualberta.ca

1 Department of Radiology and Diagnostic Imaging, University of Alberta, 8440-112 St., Edmonton, Alberta T6G 2B7, Canada

2 Department of Radiology and Diagnostic Imaging, Servier Virtual Cardiac Centre, University of Alberta, Mazankowski Alberta Heart Institute, Edmonton, Alberta, Canada male with post-traumatic respiratory failure in 1972 [1]. The most encouraging ECMO outcomes were subsequently seen in neonatal respiratory failure. Since the inception of the Extracorporeal Life Support Organization (ELSO) in 1989, approximately 98,840 patients have benefited from ECMO worldwide (41\% neonate, $23 \%$ pediatric, and $36 \%$ adult) [2]. Of the neonatal ECMO patients, $77 \%$ required respiratory
3 Department of Surgery, Division of Cardiac Surgery, University of Alberta, Stollery Children's Hospital \& Mazankowski Alberta Heart Institute, Edmonton, Alberta, Canada

4 Department of Anesthesiology and Pain Medicine, University of Alberta, Edmonton, Alberta, Canada

5 Department of Pediatrics, Pediatric Cardiac Intensive Care, Stollery Children's Hospital, University of Alberta, Edmonton, Alberta, Canada 
support, $19 \%$ cardiac support and $4 \%$ cardiopulmonary support. Of the pediatric ECMO patients, $45 \%$ needed cardiac support, $39 \%$ respiratory support and $16 \%$ cardiopulmonary support. These trends are changing as better therapy has become available for neonatal respiratory failure and as there is increasing use of ECMO for pediatric and adult cardiac failure.

There are several goals in pediatric ECMO use. The bridge to recovery strategy replaces the heart and/or lung functions until the host's organs recover [3]. In the bridge to decision strategy, ECMO stabilizes the patient to buy time for assessing end organ function and re-evaluating treatment goals [4].

This pictorial review will help pediatric radiologists understand the basics of ECMO in the pediatric population from both clinical and radiology perspectives.

\section{The ECMO circuit}

Blood is removed from and returned to the patient via cannulae that connect to the ECMO circuit (Fig. 1). The cannulae and their ports are visible on radiograph (Fig. 2). Deoxygenated blood flows from the patient via a drainage cannula located in either a large central vein or the right atrium and then into the ECMO circuit where it is circulated by a pump through the oxygenator, which is the site of $\mathrm{O}_{2} / \mathrm{CO}_{2}$ gas

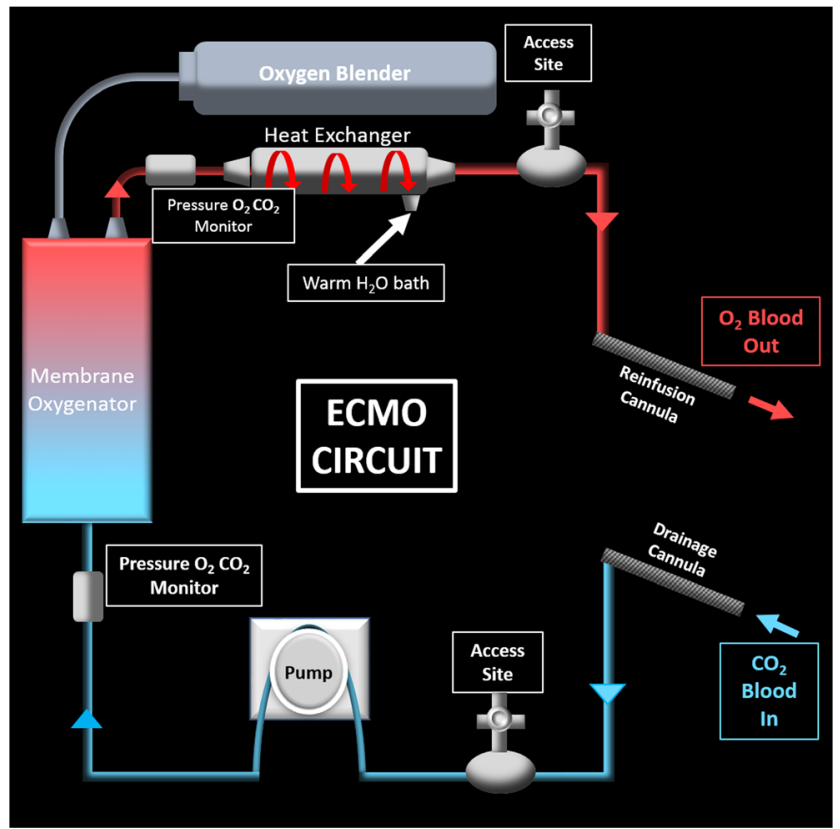

Fig. 1 Deoxygenated blood flows into the ECMO (extracorporeal membrane oxygenation) circuit via one or more cannulae. While flowing through the circuit, blood is oxygenated and the flow rate, pressure and temperature are adjusted to control blood pressure/body perfusion and body temperature (heating and cooling), respectively. Access sites allow for blood sampling as well as addition of medications, electrolytes and/or contrast to the bloodstream. The blood flows back into the patient via a separate cannula

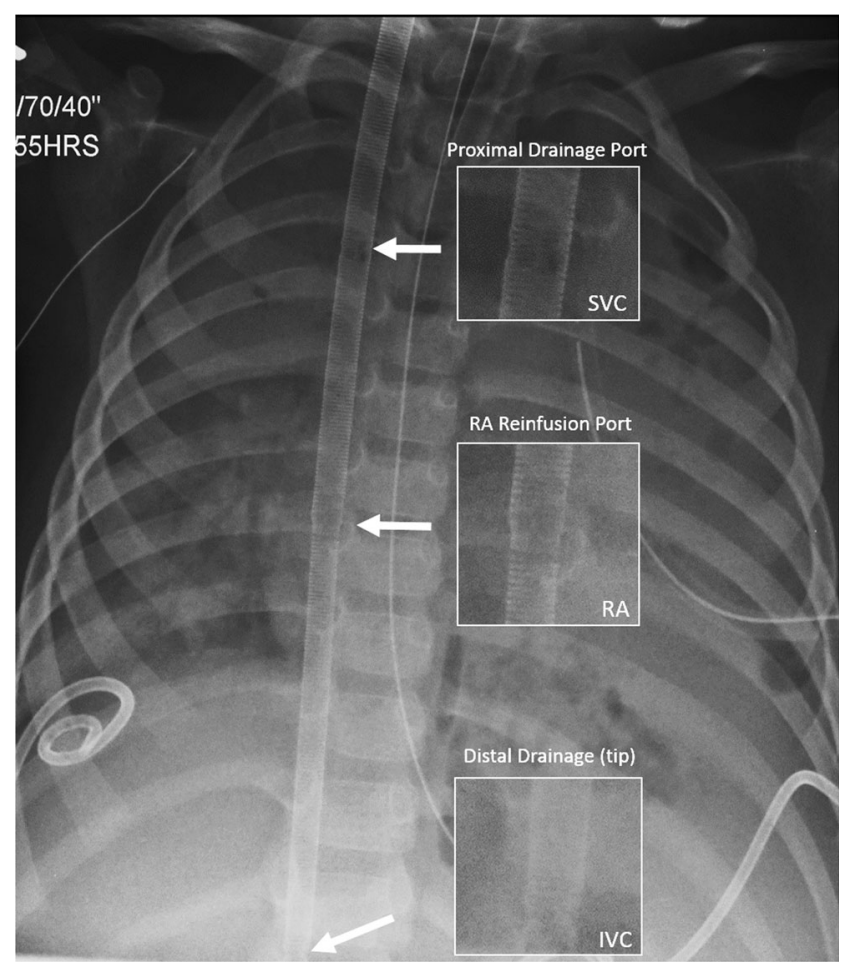

Fig. 2 Dual lumen veno-venous ECMO (extracorporeal membrane oxygenation) cannula in a 4-year-old boy with acute respiratory distress syndrome from bacterial pneumonia. This single-site access cannula has two drainage ports that move blood into the ECMO circuit (visible on radiograph and located in the mid superior vena cava [SVC] and the hepatic inferior vena cava [IVC]). Blood is reinfused into the corporeal circulation via a separate lumen in the catheter with the single reinfusion port located in the right atrium and oriented toward the tricuspid valve. $R A$ right atrium

exchange [5]. Pressures, $\mathrm{CO}_{2}, \mathrm{O}_{2}$ and flow rates are monitored and adjusted to ensure that ECMO meets the body's metabolic needs and organs are adequately perfused. When the heat exchanger is present, it adjusts blood temperature and, therefore, body temperature. This can be used in a hypothermia protocol, for example. Blood is returned to the body via the reinfusion cannula into either a large central vein or an artery depending on the ECMO mode. Medications [6], fluids, blood products and contrast can be infused and blood sampled at the access sites along the circuit.

\section{Indications}

ECMO is used with cardiac or respiratory failure refractory to conventional medical management [5]. Examples of respiratory indications include acute respiratory distress syndrome [4], bridge to lung transplant, pneumonia, status asthmaticus and congenital diaphragmatic hernia. Examples of cardiac indications include cardiogenic shock, post cardiotomy (e.g., for congenital heart disease repair), bridge to assist device [4] and extracorporeal CPR. 


\section{Clinical considerations}

Anticoagulation is necessary in all ECMO patients to avoid circuit thrombosis $[5,7]$. There is a risk of bleeding, as well as thrombosis/embolism, due to nontherapeutic anticoagulation levels.

Blood pressure is controlled by ECMO circuit flow rates, medications (e.g., vasopressors), blood products and intravenous fluids. Inadequate organ perfusion due to insufficient circuit flow or vasoplegia predisposes to ischemia. Elevated circuit flow pressures predispose to hemolysis.

Oxygenation is controlled by sweep flow and $\mathrm{FiO}_{2}$ to the membrane oxygenator. These are adjusted to optimize $\mathrm{PaO}_{2}$ to avoid oxygen toxicity and hypoxemia.

Ventilator pressures are reduced to allow for lung rest and to minimize ventilator-associated lung injury [8]. Temperature is controlled by the heat exchanger (which is variably present) to ensure normal body temperature or to maintain a hypothermia protocol.

Sedation is usually warranted during cannula placement to avoid cannula dislodgement. Pediatric patients are sedated while on ECMO for this same reason, as well as for comfort and to avoid both over-breathing the ventilator and the higher oxygen demand and higher metabolic requirements that come with increased patient activity.

\section{ECMO modes}

ECMO can be veno-venous (VV ECMO), used in respiratory failure with normal cardiac function, or veno-arterial (VA ECMO), used in cardiac or cardiopulmonary failure. VA ECMO may also be used in neonates with respiratory failure.

\section{VV ECMO}

VV ECMO is used in respiratory failure. The VV ECMO circuit oxygenates and removes $\mathrm{CO}_{2}$, replacing lung function, while the normally functioning heart continues to circulate blood through the body. VV ECMO can be further divided into two-site VV ECMO (Fig. 3) and single-site, dual-lumen VV ECMO (Fig. 4).

\section{Two-site VV ECMO}

In two-site VV ECMO, the drainage and reinfusion cannulae are placed via two different large central venous access sites. The usual pediatric configuration of two-site VV ECMO includes:

1. A reinfusion cannula in the right internal jugular vein with the radiolucent tip $4 \mathrm{~cm}$ beyond the radiopaque tip in the high to mid right atrium (Fig. 5).

2. A drainage cannula in either common femoral vein [4] with the tip at the inferior cavoatrial junction.

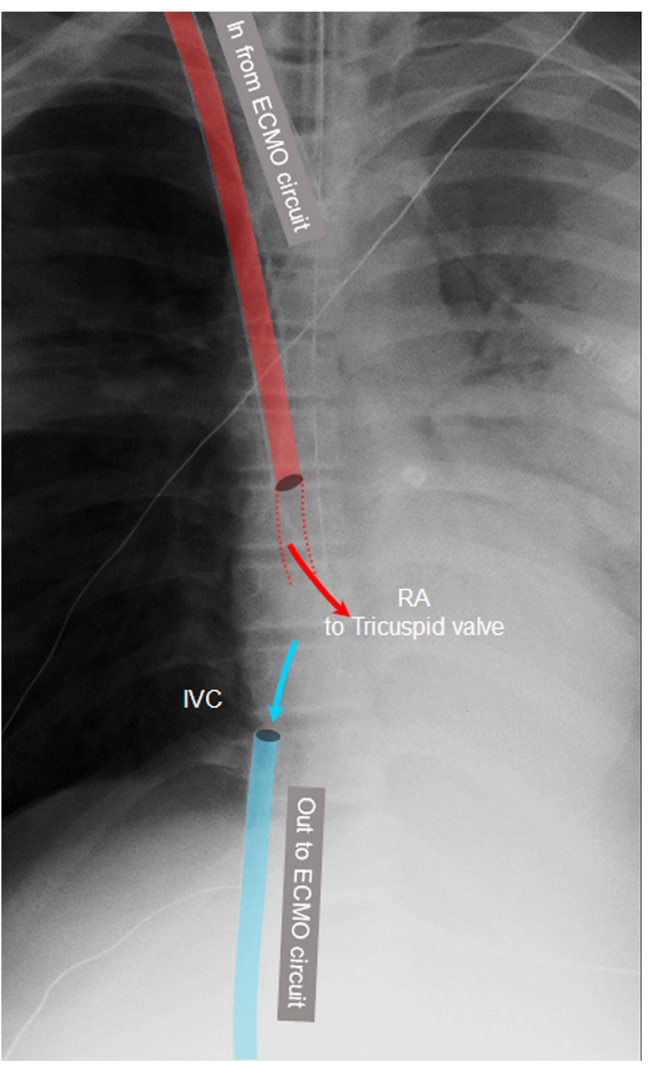

Fig. 3 Two-site veno-venous ECMO (extracorporeal membrane oxygenation) in a 13-year-old boy with acute respiratory distress syndrome from Influenza A infection. This ECMO mode includes an IVC drainage cannula (blue) and a right internal jugular vein reinfusion cannula (red). The right internal jugular vein is preferred because of its more direct course to the right atrium (RA). Again, flow from the reinfusion cannula is directed toward the tricuspid valve

Flow from the reinfusion tip is directed toward the tricuspid valve using echocardiography (Fig. 3) [9]. This promotes antegrade flow of the newly oxygenated blood into the right ventricle over admixture with venous blood in the right atrium. The greater the admixture of newly oxygenated and outgoing venous blood in the right atrium, the more oxygenated blood is taken up by the nearby drainage catheter without ever perfusing the tissues. This phenomenon is known as recirculation, and it decreases both the efficiency and effectiveness of the ECMO circuit. Cannula positioning is adjusted to minimize recirculation.

The right internal jugular vein is preferred over the left internal jugular vein approach, as it is a more direct trajectory to the right atrium. The angle of insertion on the left is more difficult and, thus, often more traumatic.

\section{Single-site double-lumen VV ECMO}

In single-site dual-lumen VV ECMO (Fig. 4), a single cannula is inserted at only one central venous access site. This is most commonly the right internal jugular vein, although the 


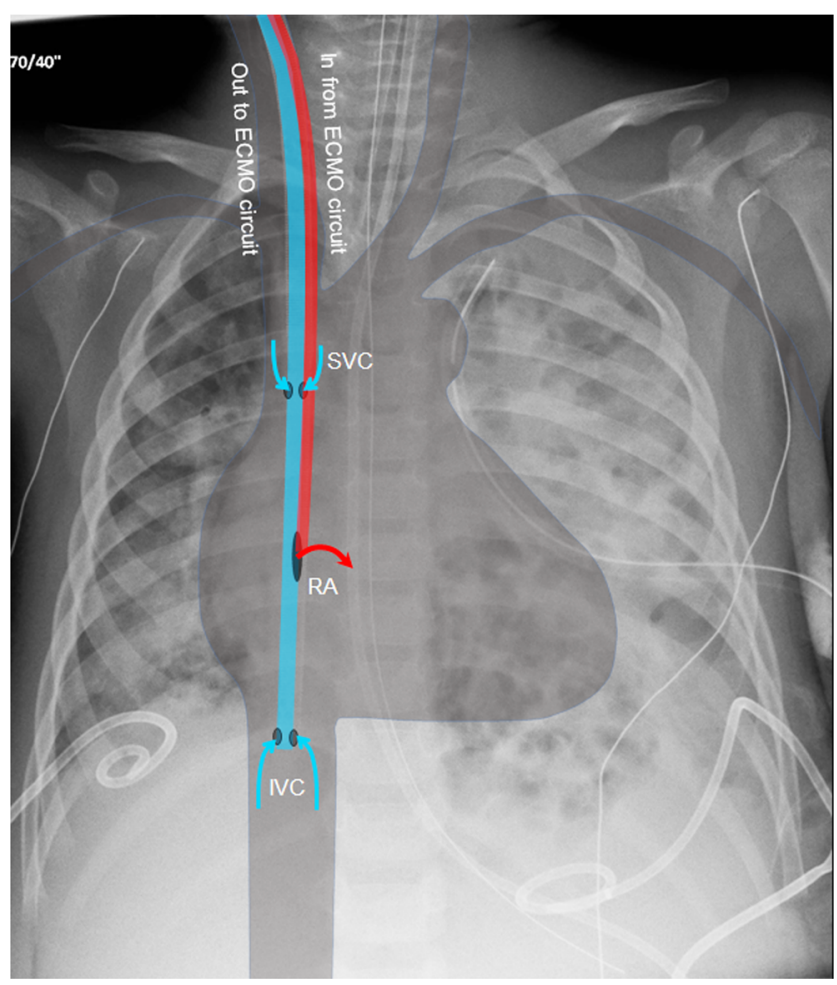

Fig. 4 Dual-lumen veno-venous ECMO (extracorporeal membrane oxygenation) cannula schematic from the same patient in Fig. 2 shows drainage (blue) and reinfusion (red) lumens in a single-site access catheter. IVC inferior vena cava, SVC superior vena cava

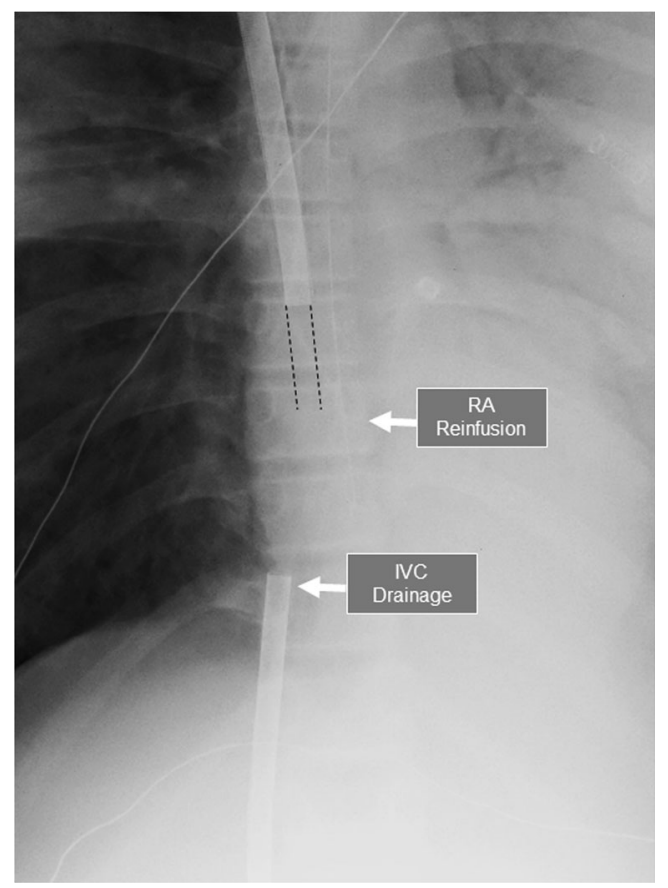

Fig. 5 Two-site veno-venous ECMO (extracorporeal membrane oxygenation) positioning in the same patient as in Fig. 3. The tip of this particular reinfusion cannula is radiolucent and is outlined by the dashed lines, extending $4 \mathrm{~cm}$ beyond the radiopaque tip and oriented toward the tricuspid valve. The inferior vena cava (IVC) drainage cannula is inserted through either common femoral vein and the tip lies at or near the inferior cavoatrial junction. $R A$ right atrium common femoral vein can be used in larger children. This cannula has two lumens, one for reinfusion and one for drainage, with associated reinfusion and drainage ports that need to be positioned properly. Dual-lumen VV ECMO is preferred to a two-site access because its single insertion site allows for fewer complications.

In dual-lumen VV ECMO, there are three ports:

\section{Two drainage ports:}

a. One in the mid superior vena cava (SVC).

b. One (the tip) in the hepatic inferior vena cava (IVC). 2. Single reinfusion port in the mid right atrium (Fig. 2).

Flow toward the tricuspid valve from the reinfusion port is confirmed by echocardiography, as with two-site VV ECMO.

In infants, the dual-lumen VV cannula is slightly different in appearance. This cannula has a radiopaque strip that delineates the catheter segment containing multiple right atrial reinfusion ports (Fig. 6). These ports are still centered in the right atrium and oriented toward the tricuspid valve. In this case, the drainage port is located in the catheter tip, which is situated in the hepatic IVC.

In neonates and small children who are not yet walking (and usually weigh less than $10 \mathrm{~kg}$ ), the common femoral

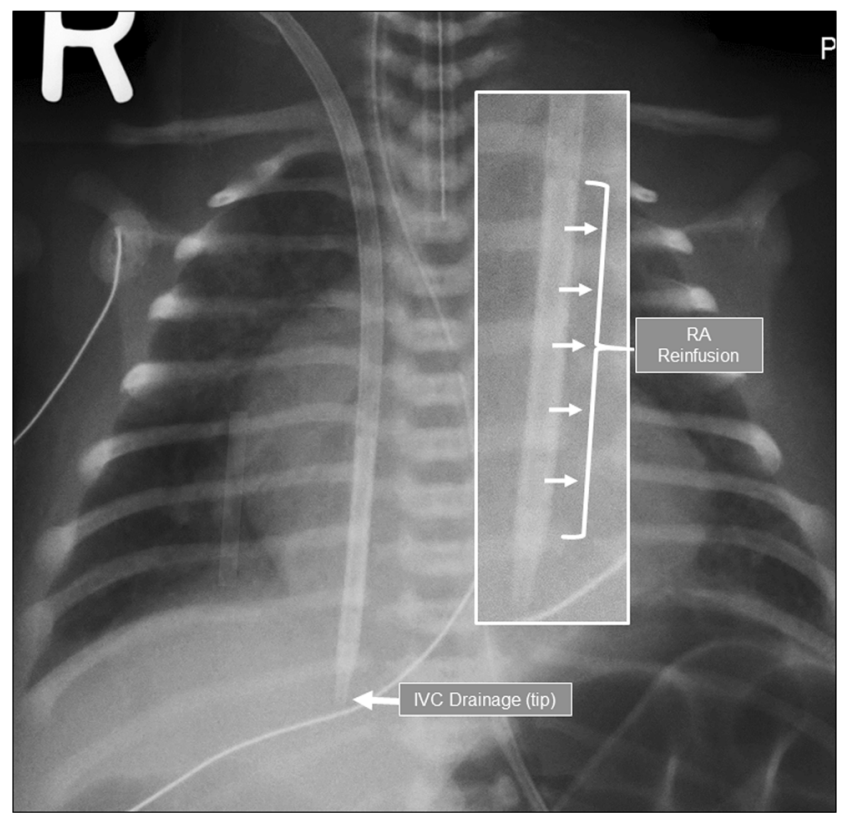

Fig. 6 Dual-lumen veno-venous (VV) ECMO (extracorporeal membrane oxygenation) in a 14-month-old boy with critical aortic stenosis and hypoplastic left heart. This is a different version of a dual-lumen VV ECMO cannula that has a radiopaque strip along the distal portion that contains multiple reinfusion ports centered in the right atrium and oriented toward the tricuspid valve. In this catheter, the drainage port is at the catheter tip and located in the hepatic inferior vena cava (IVC). It moves blood to the ECMO circuit via a separate lumen. $R A$ right atrium 
artery and vein access sites are avoided for any ECMO cannulation. This is because the common femoral artery and vein are still very small in caliber and cannot yet accommodate the large-bore ECMO cannulae, thus risking vessel occlusion and damage $[5,10]$.

\section{VA ECMO}

In addition to oxygenation and the removal of $\mathrm{CO}_{2}$ from the blood, VA ECMO pumps the blood through the body by virtue of blood reinfusion directly into the arterial system. VA ECMO is used when any component of cardiac failure is present, regardless of respiratory status. In neonates with respiratory failure, VA ECMO may also be used instead of two-site VV ECMO.

\section{Peripheral VA ECMO}

In this configuration, venous and arterial cannulae are placed in their respective large peripheral vessels. The most common approach (Fig. 7) includes:

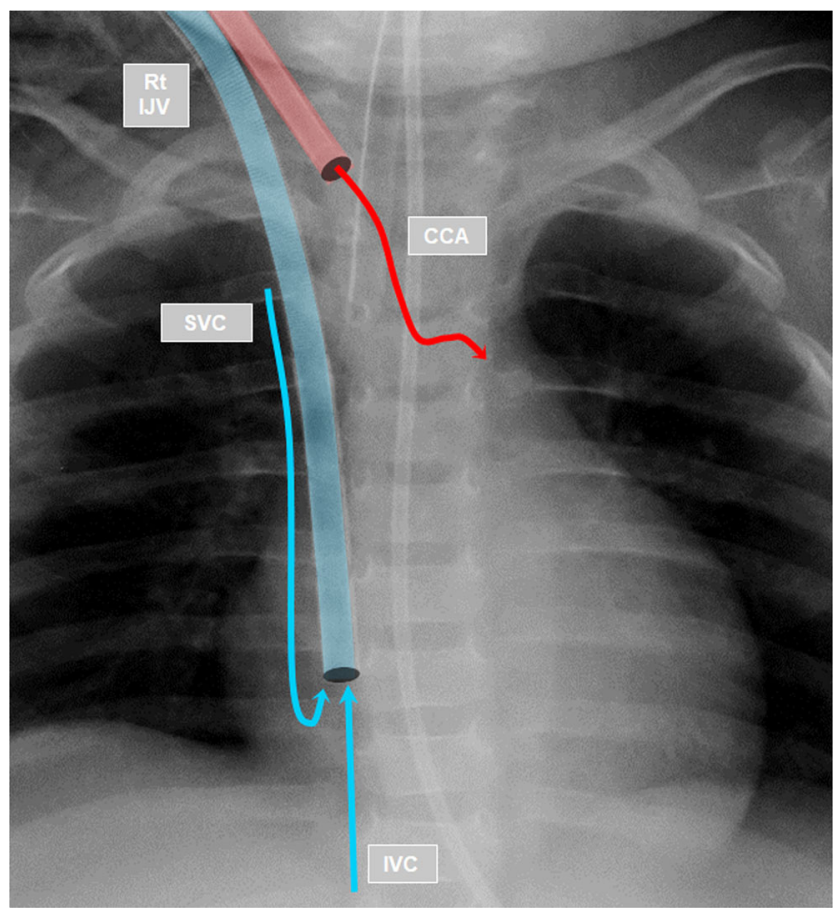

Fig. 7 Peripheral veno-arterial (VA) ECMO (extracorporeal membrane oxygenation) in a 21-month-old girl with dilated cardiomyopathy. The access sites of both cannulae are in the periphery at the level of the right lower neck, rather than directly in the heart, as is the case with central VA ECMO. The alternative peripheral VA access site is in the groin, which is less favored in children. The venous cannula (blue) removes blood from the right atrium/hepatic inferior vena cava (IVC) region into the ECMO circuit. The arterial cannula (red) reinfuses blood into the arterial circulation via the common carotid artery (CCA). Rt IJV right internal jugular vein, $S V C$ superior vena cava
1. Drainage cannula in the right internal jugular vein that collects deoxygenated blood via its tip in the low right atrium/hepatic IVC (Fig. 8) [3].

2. Reinfusion cannula in the right common carotid artery. The cannula tip is radiopaque and should be in the mid to distal common carotid artery, approximately $4 \mathrm{~cm}$ from the skin insertion site.

Less commonly, a groin access can be used that includes drainage from either common femoral vein (with cannula tip near the inferior cavoatrial junction) and reinfusion via the common femoral artery (with cannula tip in the common femoral or distal external iliac artery, $4 \mathrm{~cm}$ from the skin insertion site). A combined groin and neck configuration is another, even less commonly used option. Again, use of common femoral vein/ common femoral artery should be avoided in small children.

Cannula position is adjusted based on radiographic measurements. While arterial cannulae are fairly consistent in appearance and tend not to have radiolucent tips, the singlelumen right internal jugular vein cannulae used for venous drainage in VA ECMO and for reinfusion in VV ECMO vary widely in appearance and often have radiolucent tips (Fig. 9). Some venous cannulae have a radiopaque dot or BB at the tip of the cannula, other cannulae have a $4-\mathrm{cm}$ radiolucent segment extending beyond the radiopaque tip with no marker.

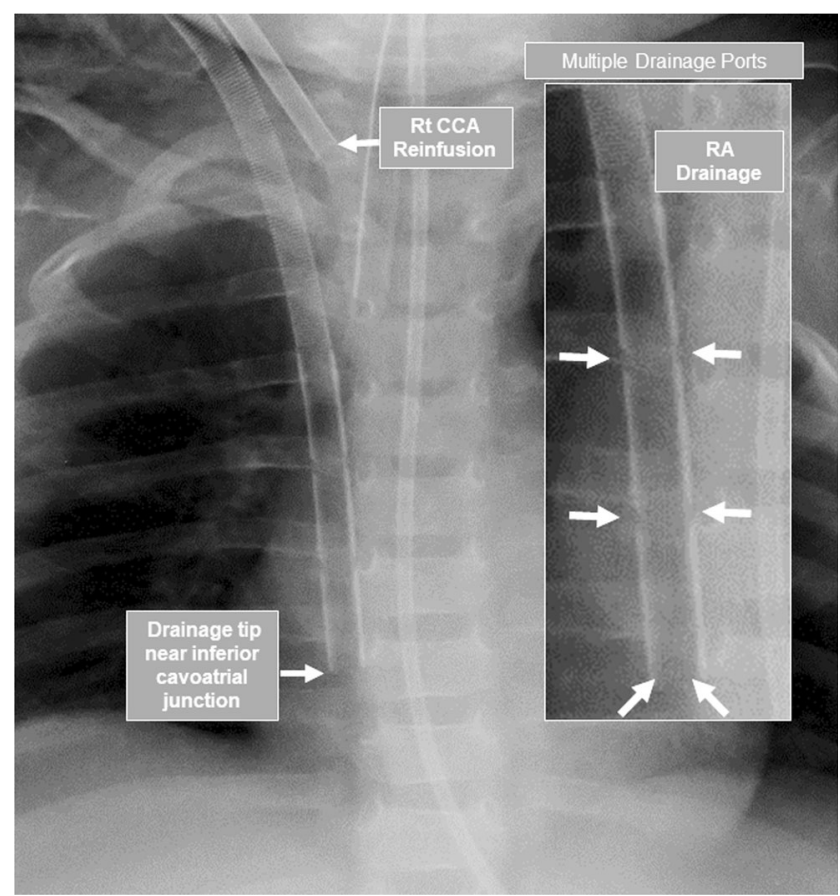

Fig. 8 Positioning of peripheral veno-arterial ECMO (extracorporeal membrane oxygenation) cannulae in the same patient as in Fig. 7. This is another variant in the appearance of the drainage cannula with the tip near the inferior cavoatrial junction as expected and multiple drainage ports centered in the right atrium (RA). The reinfusion cannula is located in the right common carotid artery (Rt CCA) with the visible tip located approximately $4 \mathrm{~cm}$ below the skin entry site in the lower neck 


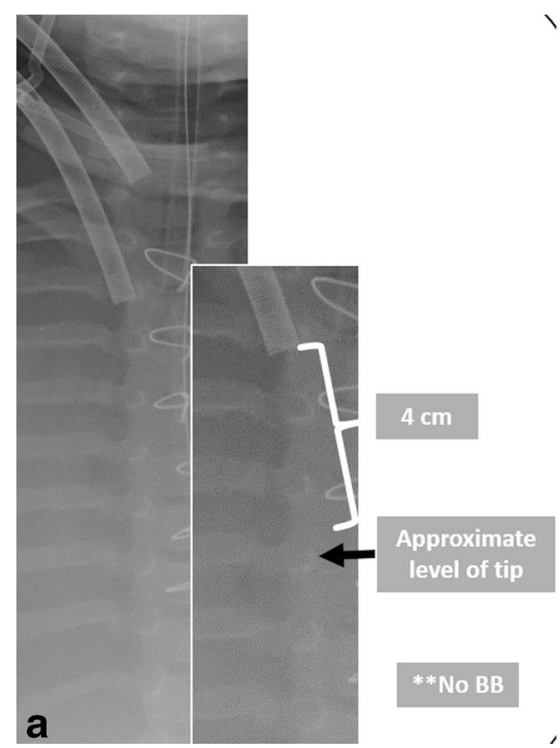

b

Fig. 9 Venous cannula variants in peripheral veno-arterial ECMO (extracorporeal membrane oxygenation) in a 3-year-old boy status post corrected transposition of the great arteries (a), 21-year-old woman with dilated cardiomyopathy (b), and 1-day-old girl with septic shock (c). a

The desired location of the venous cannula tip is always in the low right atrium/hepatic IVC, however, regardless of appearance.

\section{Central VA ECMO}

In central VA ECMO, a sternotomy is performed and the cannulae are placed transthoracically into the respective vessel/

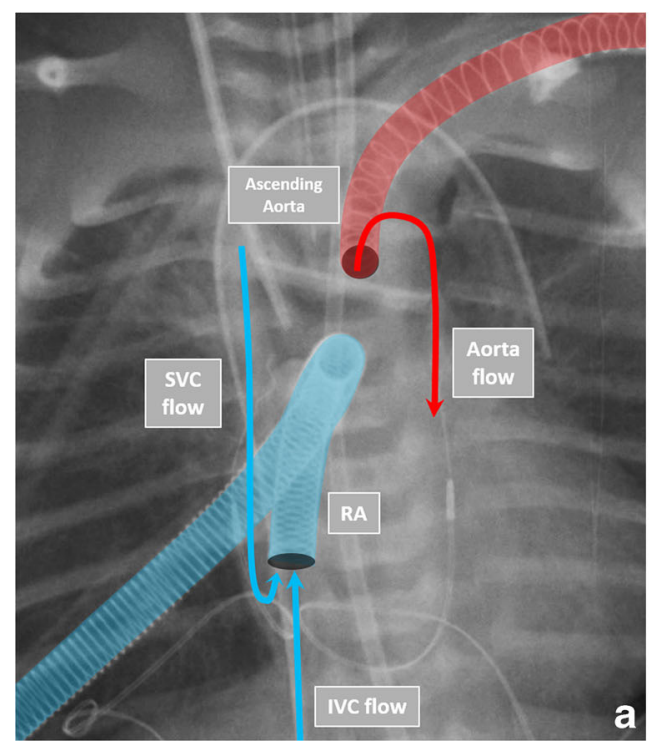

Fig. 10 Central veno-arterial (VA) ECMO (extracorporeal membrane oxygenation) in a 20-day-old girl with ectopia cordis and Pentology of Cantrell. a After sternotomy, the drainage (blue) and reinfusion (red) cannulae are surgically placed directly into the right atrium (RA) and ascending aorta, respectively. b The sternum is often left open with central VA ECMO (with clear plastic adhesive drape placed over top)

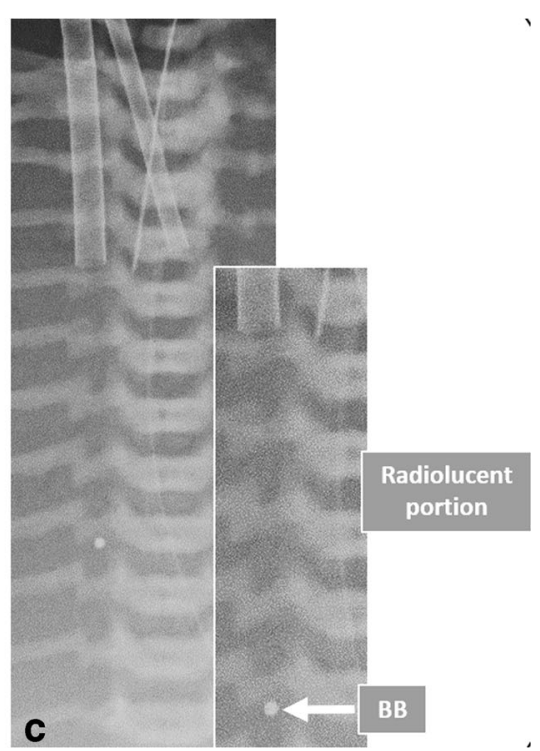

Radiolucent tip is not seen and typically extends $4 \mathrm{~cm}$ beyond the radiopaque tip with no $\mathrm{BB}$ in place. b Radiopaque cannula tip with multiple side ports (arrows) for drainage. c Radiolucent segment of distal cannula this time with tip demarcated by radiopaque BB

heart chamber. The venous drainage cannula is sutured directly into the right atrium and the arterial reinfusion cannula is sutured directly into the ascending aorta (Fig. 10). This procedure is more invasive with a higher risk of hemorrhage at the insertion sites [11].

Because ECMO patients are anticoagulated, bleeding is the most common complication. The cannula insertion sites are the most common site of bleeding [8]. In the

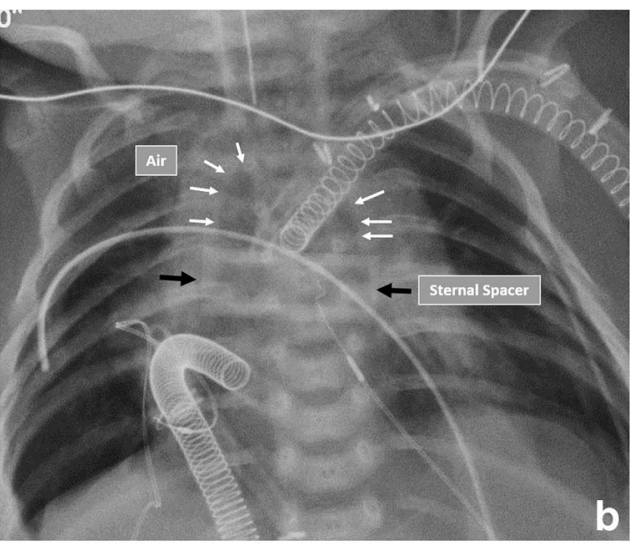

because bleeding at the access site is the most common complication of ECMO and can thus be easily detected. On radiographs, you will invariably see the sternal spacer (black arrows) in place, and often mediastinal air (white arrows), if the mediastinum has been recently opened. IVC inferior vena cava, $S V C$ superior vena cava 
setting of VA ECMO, since bleeding complications are most likely to occur in the operative bed, the sternum is left open with a transparent adhesive drape in place. This allows for easy detection of and access to hemorrhage at the insertion sites without having to repeat the sternotomy. The sternotomy is kept open by the sternal spacer device, which can be seen on radiograph (Fig. 10). If the site has been open recently, mediastinal air may also be seen on the radiograph (Fig. 10).

\section{Accessory venous/drainage cannulae}

One or more accessory venous drainage cannulae can be added to any VA or VV ECMO circuit (Fig. 11). These increase the volume of deoxygenated blood removed from the patient into the ECMO circuit, which thereby increases the amount of oxygenated blood returning to the patient. The most common accessory drainage cannula is placed in a cephalad/retrograde orientation within the right internal

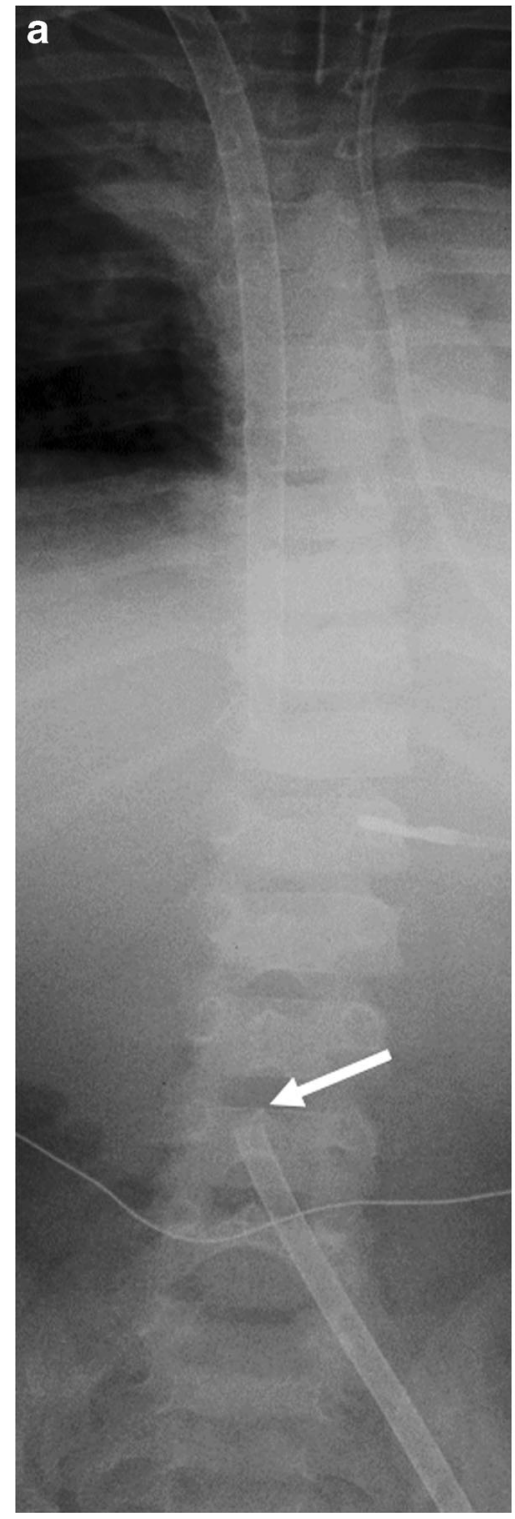

Fig. 11 Examples of accessory venous cannulae in a 5-year-old boy with adenovirus pneumonia (a), a 19-month-old girl with repaired subaortic membrane (b), a 2.5-year-old girl with acute lymphoblastic leukemia, febrile neutropenia, and acute respiratory distress syndrome (c), a 21month-old girl with trisomy 21 and cardiac arrest status post atrioventricular septal defect repair (c), and a 4-month-old girl with coarctation, partial anomalous pulmonary venous return, and anomalous left coronary artery arising from the pulmonary artery (ALCAPA) status post ALCAPA repair (d). a Single-site dual-lumen veno-venous ECMO (extracorporeal membrane oxygenation) in the right internal jugular vein with accessory drainage cannula in the left common femoral vein (arrow); (b) peripheral veno-arterial (VA) ECMO in the neck with added drainage cannula through the interatrial septum into the left atrium (arrow); (c) peripheral VA ECMO with accessory cephalad drainage cannula in the right internal jugular vein (arrow), and (d) central VA ECMO with accessory drainage cannula in the left atrial appendage (arrow) 
jugular vein, just above the access level for the primary, caudally directed right internal jugular vein cannula.

Accessory venous/drainage cannulae can also be positioned with the tip in any large central vein not being used by the primary drainage cannula (common femoral vein, internal jugular vein, inferior vena cava and superior vena cava). In VA ECMO, the accessory drainage cannula may be placed with the tip in the left atrium, decompressing elevated left atrial pressures that have developed secondary to left ventricle systolic dysfunction. The associated pulmonary edema improves as well, as detailed below.

It should be noted that the position of any cannula placed in the neck can be altered by a change in the position of the head or neck, just as with the endotracheal tube. Subtle changes in position may not be apparent on radiograph and will often need confirmation via echocardiogram.

\section{Normal lung changes}

Pediatric patients are typically intubated per ECMO protocol. After ECMO initiation, there is often a predictable pattern of change in the radiographic appearance of the lungs over time (Fig. 12). The lungs show progressive opacification on radiograph, often to the point of complete whiteout, followed by eventual complete or nearcomplete resolution. The timing of these changes is highly variable, but opacities appear generally within $24 \mathrm{~h}$ of ECMO placement and can last only a few days or as long as 4 to 6 weeks, depending on the patient's pathophysiology. If significant opacification persists beyond that time, there is usually a component of severe or irreversible lung pathology and the prognosis is poor.

The exact etiology of these changes is multifactorial. The low ventilator pressures and $\mathrm{FiO}_{2}$ needed to
Fig. 12 Typical progression in the radiographic appearance of the lungs in a 3-year-old boy recently placed on veno-arterial ECMO (extracorporeal membrane oxygenation) intraoperatively during correction of transposition of the great arteries. Failure to resolve opacities at least partially after several weeks of ECMO usually indicates a poor prognosis
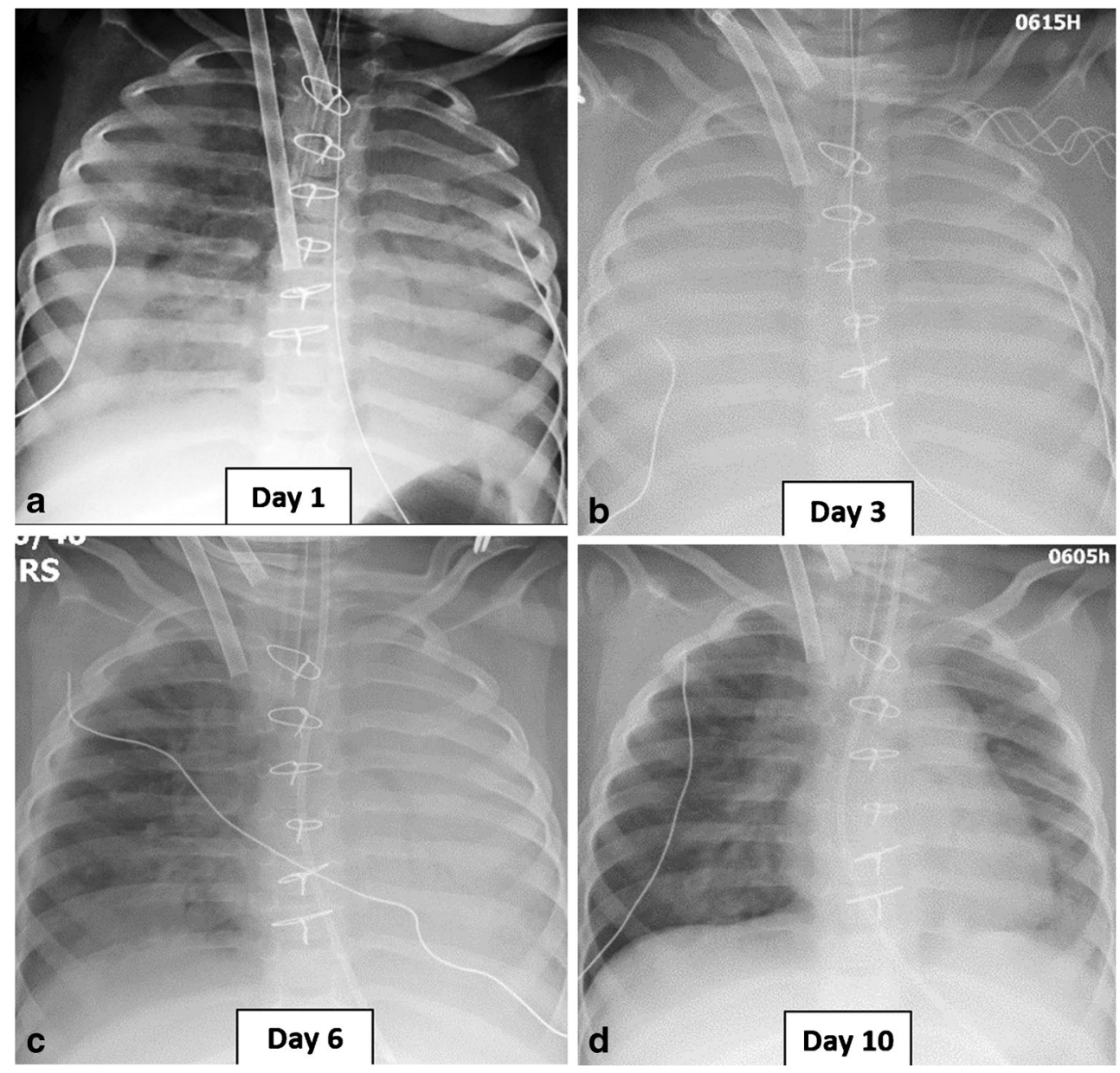
achieve lung rest in ECMO patients result in varying degrees of widespread atelectasis that partly contributes to the lung opacification [8]. It is also known that after initiation of ECMO, the patient undergoes a systemic inflammatory immune response toward the exogenous antigens from the ECMO circuit [12]. This causes widespread fluid retention throughout the body, including variable pulmonary edema that is an additional contributor to the lung opacification on radiograph. These changes are more common with VV ECMO.

Patients requiring VA ECMO have cardiac dysfunction and often left ventricular systolic dysfunction. The increased afterload caused by the addition of the arterial cannula may put further strain on the heart. Both factors combine to cause elevated left heart pressures and severe pulmonary edema [13]. When an accessory drainage cannula is placed in the left atrium, the pulmonary edema often improves or resolves completely. This phenomenon is specific to VA ECMO.

\section{Contrast-enhanced CT in ECMO}

By adding the ECMO circuit and its pump in series to the native circulatory system, hemodynamics are markedly altered and contrast-enhanced CT becomes complex. Consideration must be given to many factors, including flow dynamics, location of cannulae, injection point, cardiac function and flow rates within the ECMO circuit.

The faster the ECMO flow rate, the more the native hemodynamics are altered and the less predictable the behavior of the contrast bolus. Therefore, no matter what type of ECMO circuit is in place, the ECMO flow rate should be decreased to the lowest rate allowable or even momentarily stopped, if possible. Careful consultation with the care team is required on a case-bycase basis to determine patient candidacy.

With a diminished ECMO flow rate, the flow through the drainage cannula will be diminished. Thus, there is less likely to be siphoning off of contrast from the right atrium via the drainage cannula in either VA or VV ECMO [14].

In the setting of VA ECMO, a diminished flow rate will minimize the cardiac bypass effect, thus maximizing opacification of the heart and pulmonary artery. Without a diminished flow rate, the ascending aorta will only fill in a retrograde fashion, resulting in admixture artifacts and the need for a delayed scan. The heart and pulmonary arteries will be opacified poorly or not at all. If anticipated, this effect can be offset by increasing the volume of contrast administered, decreasing the contrast infusion rate and increasing the scan delay to optimize contrast opacification.

With some planning, contrast may be injected via a peripheral vein, a central venous catheter with tip in the right atrium, or via the ECMO circuit itself, ultimately entering into the reinfusion cannula. Injection directly into the ECMO circuit better assures a more instantaneous contrast bolus [14].

\section{Complications}

Common ECMO complications are vascular, including bleeding, thrombosis, embolism, ischemia and vessel damage. Additional complications are equipment-related, such as cannula kinking or fracture, cannula malposition (Fig. 13) and equipment failure.

Bleeding is the most common complication, and the cannula insertion sites are the most common sites of bleeding. The second most common site of bleeding is a recent operative site. Ultimately, however, spontaneous hemorrhage can happen anywhere. An intracranial hemorrhage is one of the most concerning sites for a bleed [15] (Fig. 14). Large volume hemorrhage, as with retroperitoneal hematoma (Fig. 15) and hemoperitoneum (Fig. 16), is also of concern.

Because of the significant prognostic implications of an intracranial bleed, as well as the overall high likelihood of hemorrhage occurring in ECMO patients, the Extracorporeal Life Support Organization (ELSO) recommends that infant patients be closely monitored with head US when possible [16]. The protocol suggested in the ELSO guidelines includes performing head US every $24 \mathrm{~h}$ for the first 5 days of ECMO, if the patient is stable. If the patient is unstable with regard to coagulation or hemodynamic status, a daily head US is recommended until stabilized. If a bleed is detected, the recommendations vary with the severity of the bleed. If there is a small intracranial bleed, head US twice daily should be considered until coagulation parameters are optimized. If it is a small to moderate but growing hemorrhage, a concerted effort should be made to wean from ECMO so as to end the need for anticoagulation. If there is a large intraparenchymal hemorrhage, ECMO withdrawal is indicated due to the poor prognosis (Fig. 17). 

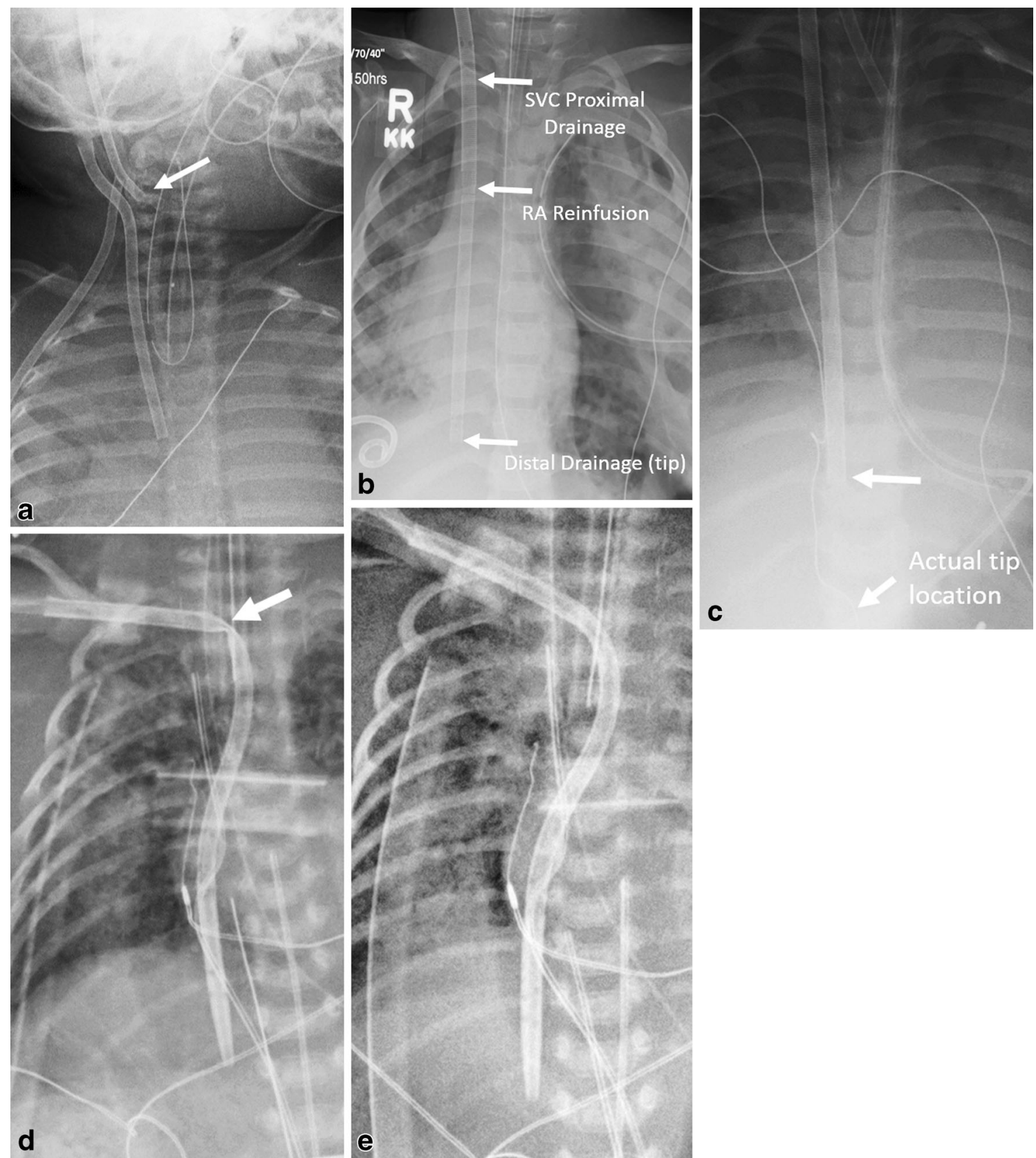

Fig. 13 Cannula malposition and kinking. a A 3-month-old girl with truncus arteriosus: Veno-arterial (VA) ECMO (extracorporeal membrane oxygenation) arterial cannula is too high: the tip (arrow) is less than 3 $4 \mathrm{~cm}$ from the insertion point into the common carotid artery. b An illfitting dual-lumen veno-venous (VV) catheter in a 4-year-old boy with bacterial pneumonia and respiratory failure. Note that only the inferior vena cava (IVC) tip (lowest arrow) is in the appropriate position in the hepatic IVC. The two more proximal ports are too high, however. c A 2.5-

Thromboembolism, another fairly common complication, can arise pre-ECMO from cardiac dysfunction (cardiogenic emboli), during ECMO (embolus year-old boy with bleach injury to body complicated by respiratory distress and cardiac arrest: The VA ECMO venous cannula tip (arrow) is too low: far below the low-right atrium, particularly when taking into account the radiolucent portion of the tip that is near the level of the renal veins. d An 8-week-old premature infant born at 28 weeks with transposition of the great arteries status post surgical correction: Kinked (arrow) dual-lumen VV catheter and (e) corrected dual-lumen VV catheter in the same patient. $R A$ right atrium, $S V C$ superior vena cava

from the circuit tubing or the heart) or with decannulation (vessel injury or shedding of embolus from the cannula) (Fig. 18). 


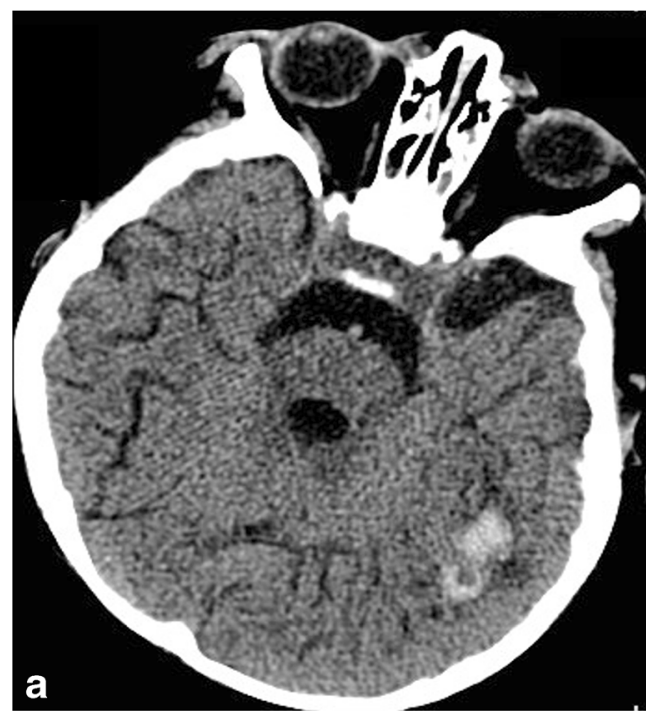

Fig. 14 Intracranial hemorrhage is one of the most feared complications in any ECMO (extracorporeal membrane oxygenation) patient. Bleeding can range from a small, single-site bleed such as the focus of intraparenchymal hemorrhage shown in a 2-year-old girl on ECMO for acute respiratory distress syndrome from pneumonia (a) to the extensive

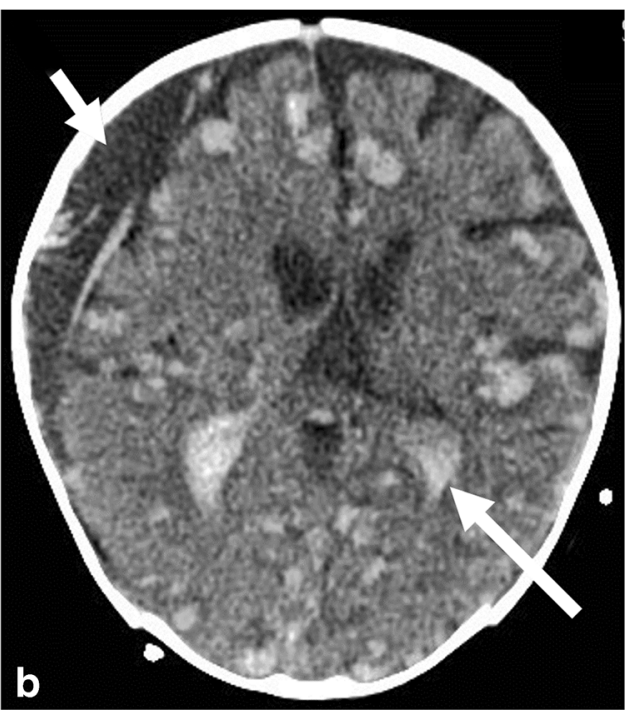

hemorrhage shown in a 6-month-old girl on ECMO for myocarditis with cardiac arrest (b), including intraventricular hemorrhage (long arrow), multifocal intraparenchymal hemorrhage, and subdural hematoma with mass effect (short arrow)
Stroke and global brain hypoxia may also occur in the setting of ECMO (Fig. 19). Several factors predispose patients to global brain ischemia [15]:

1. Prior to ECMO placement, there may be prolonged hypoxia related to the patient's state of cardiac or pulmonary failure.
2. During cannula placement, the patient is at risk for prolonged disruption of adequate blood flow.

3. While on ECMO, the circuit may not maintain adequate mean arterial pressures.

The combination of the innately small vessels of a pediatric patient and the large caliber of ECMO cannulae

Fig. 15 Spontaneous hemorrhage in a 13-year-old boy on ECMO (extracorporeal membrane oxygenation) for acute respiratory distress syndrome and sepsis from Hantavirus. Hemorrhage can happen anywhere in these anticoagulated patients with the large volume bleeds being of more concern. Axial (a) and sagittal (b) non-contrast CT images show a spontaneous retroperitoneal hematoma (arrows). Note the hematocrit level within the collection
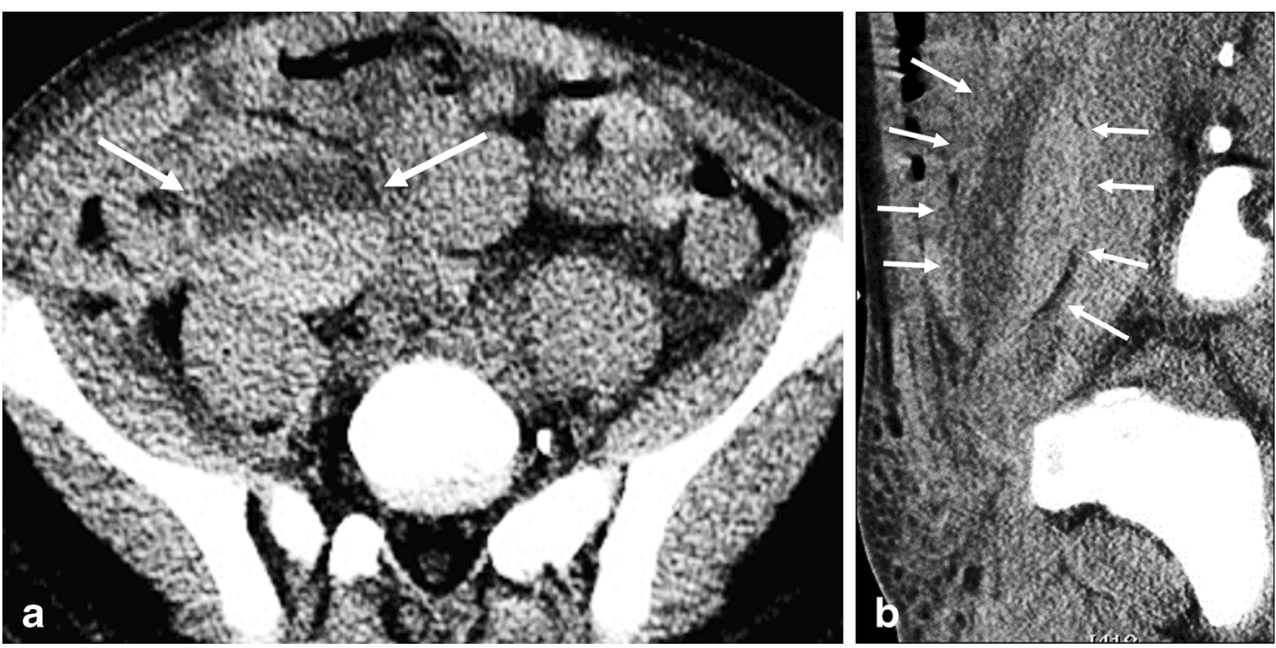


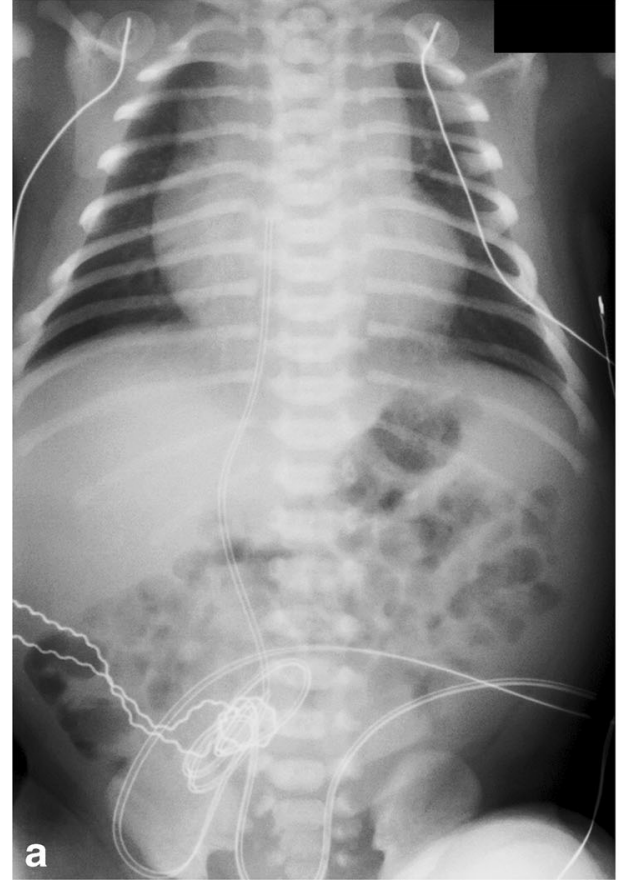

Fig. 16 Hemoperitoneum in a neonate with critical aortic stenosis and hypoplastic left heart on veno-arterial (VA) ECMO (extracorporeal membrane oxygenation) acutely decompensates after UAC repositioning. a Baseline anteroposterior (AP) radiograph predecompensation shows high umbilical venous catheter (UVC) and very low UAC but is otherwise unremarkable. b An AP abdominal radiograph obtained during an acute decompensation event shows that the patient is

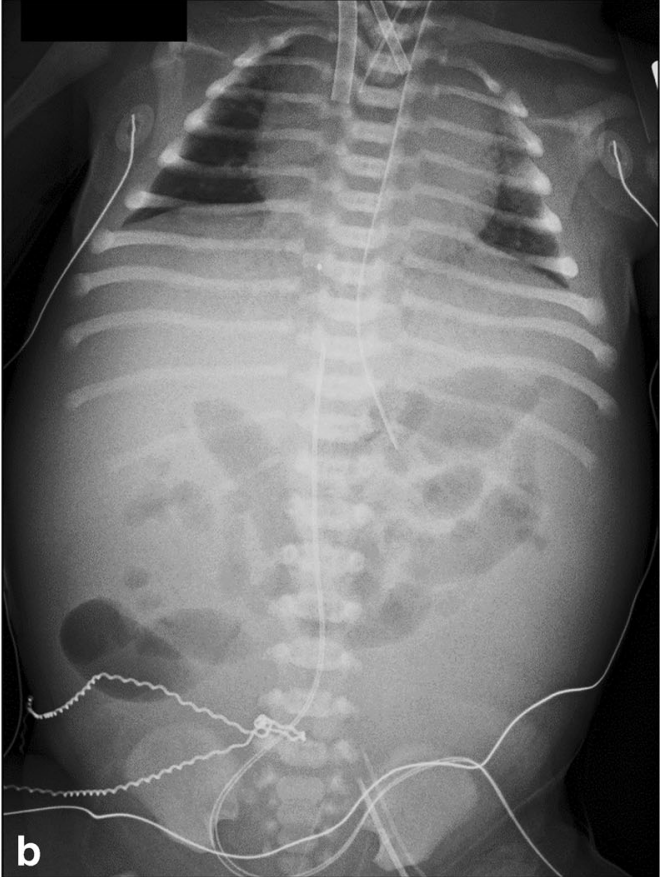

now on peripheral VA ECMO with interval repositioning of the UAC (UVC removed). There is new abdominal distension and centralization of bowel loops, indicating a new intraperitoneal fluid. Large volume hemoperitoneum from umbilical arterial catheter repositioning was diagnosed at surgery and was thought to be related to anticoagulation from ECMO

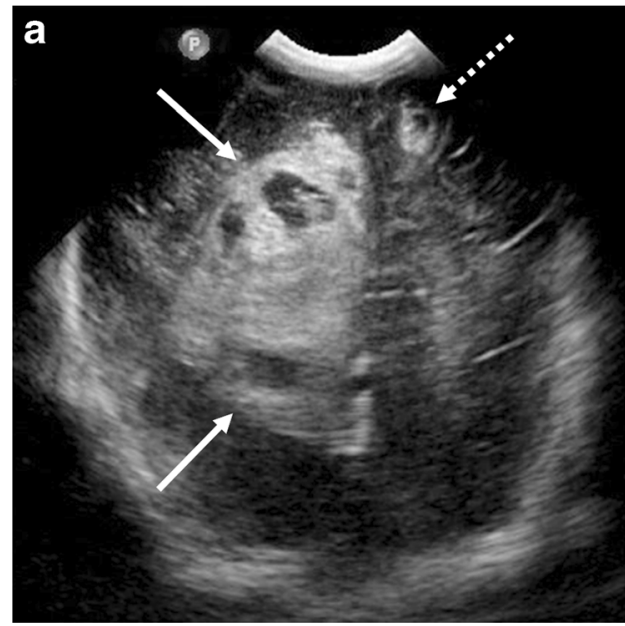

Fig. 17 Brain imaging in an 8-week-old boy on ECMO (extracorporeal membrane oxygenation) status post repair of transposition of the great arteries with acute onset of fixed pupils. A coronal image from the urgent head US (a) shows a large, heterogeneously echogenic area with cystic change in the right occipitoparietal lobe (solid arrows) and a small echogenic focus with cystic change in the subcortical white matter of

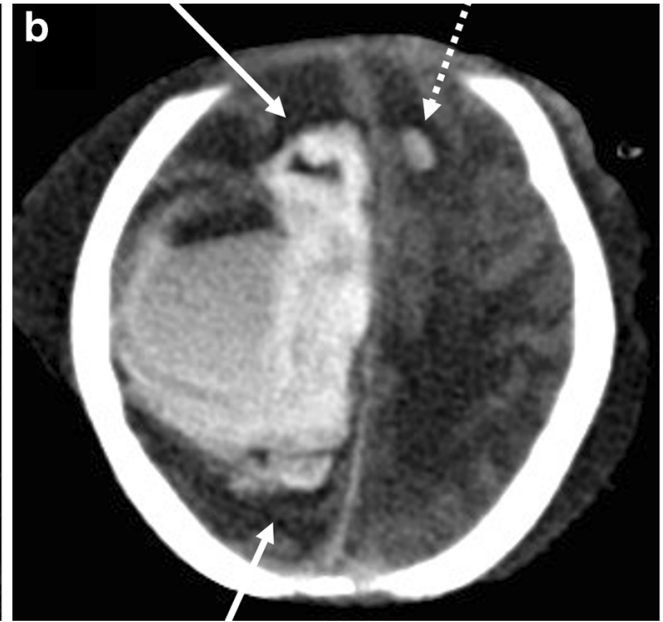

the left parietal lobe (dashed arrow), both concerning for acute hemorrhage. An axial slice from the non-contrast CT (b) performed the following day shows the same large right (solid arrows) and tiny left (dashed arrow) parietal intraparenchymal hemorrhages. The hemorrhage was followed by head US 
Fig. 18 Thromboembolic infarcts. Diffusion-weighted MR image (a) show multiple bilateral embolic infarcts in a 13-year-old boy on veno-arterial (VA) ECMO (extracorporeal membrane oxygenation) for sepsis who developed new encephalopathy a few hours after ECMO

decannulation. b Non-contrast CT shows left middle cerebral artery infarct in a 3-year-old girl on VA ECMO for status asthmaticus with cardiac arrest
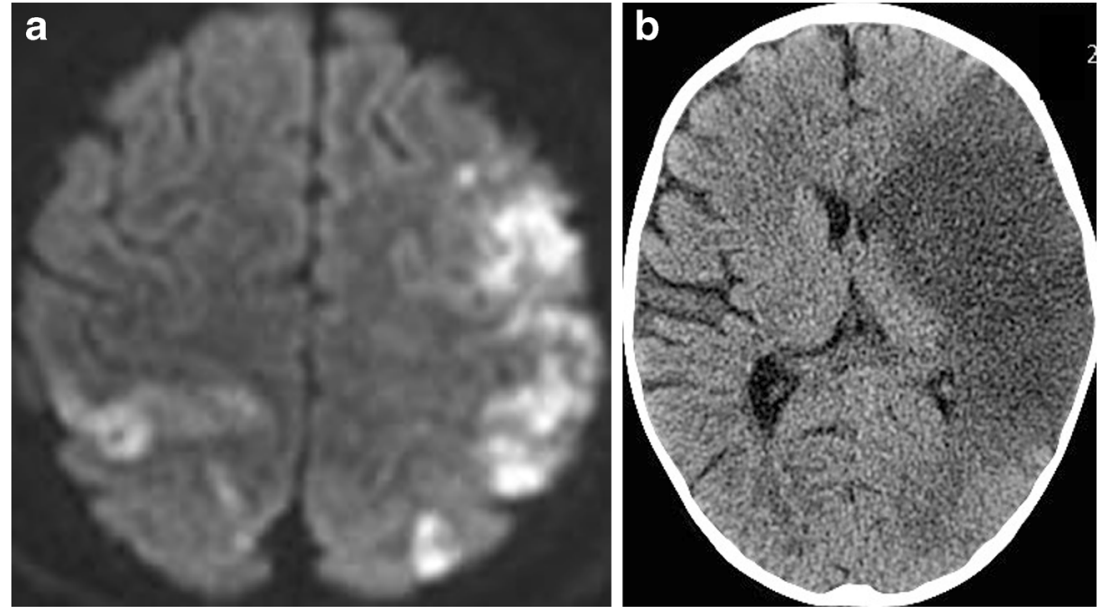

places the patient at risk for vascular injury such as arterial dissection (Fig. 20), which is a more rare complication. This same combination of a large-caliber cannula relative to small vessel lumen can cause luminal occlusion and resulting ischemia of brain in cases of common carotid artery access and lower limb in cases of common femoral artery access. Amputation may be warranted in cases of severe limb ischemia.

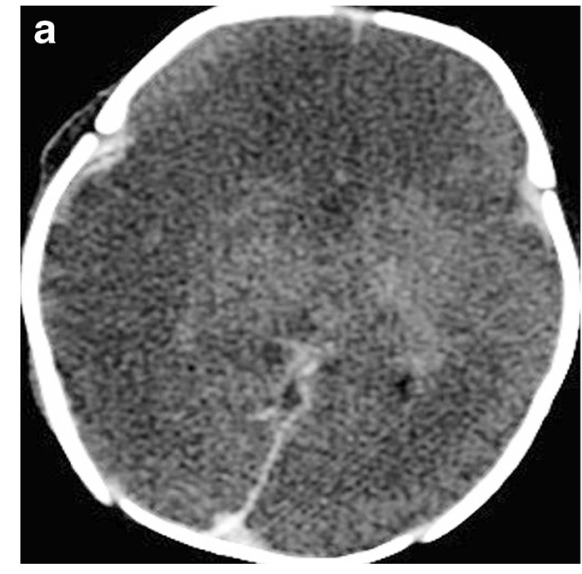

Fig. 19 Brain ischemia. a Non-contrast head CT in a 7-week-old girl on ECMO (extracorporeal membrane oxygenation) for septic shock shows diffuse cerebral ischemia, represented by edema, diffuse loss of greywhite differentiation, midline shift and sulcal/ventricle effacement. This occurred within $48 \mathrm{~h}$ of starting ECMO for septic shock and was likely related to the low pressures incurred during ECMO cannulae placement.

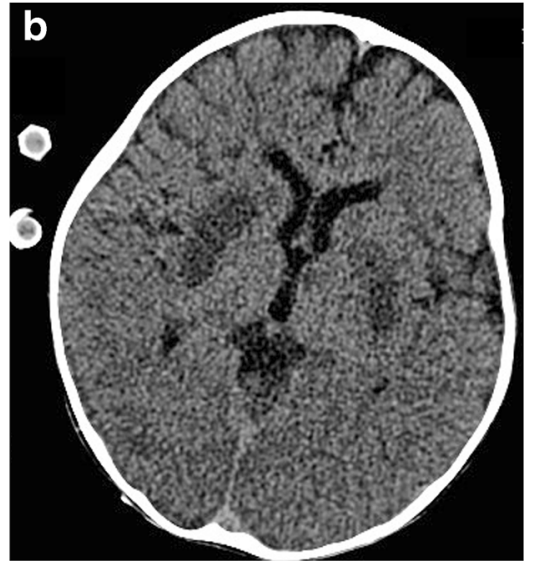

b Non-contrast head CT in a 19-month-old girl with subaortic stenosis and cardiac arrest obtained after the patient was placed on veno-arterial ECMO. Infarcts are noted in the putamina, in keeping with acute, near total asphyxia caused by the combination of low flow at ECMO initiation superimposed on baseline poor perfusion 

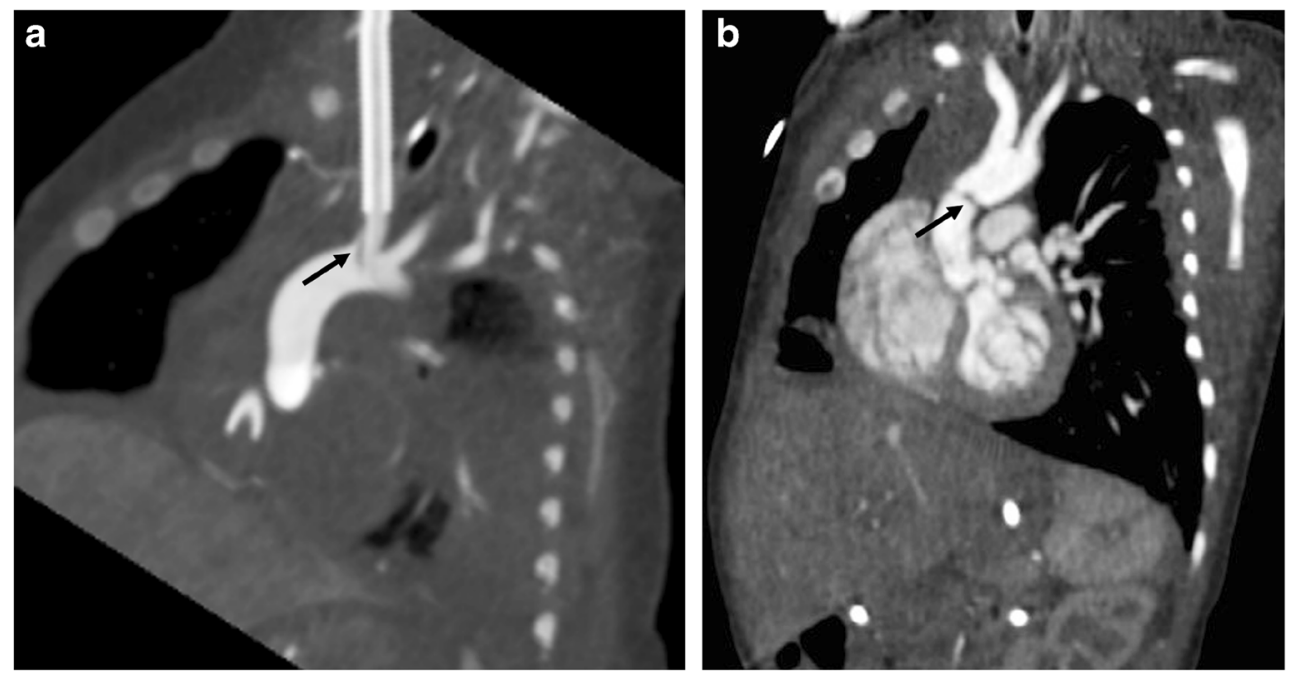

Fig. 20 Arterial dissection. CT angiogram of the chest (a) for a term neonate with congenital heart disease that was cannulated for venoarterial (VA) ECMO (extracorporeal membrane oxygenation). Contrast was injected via the arterial cannula (note that the aorta is opacified while the heart is not) and the arrow shows the dissection flap in the right common carotid artery that extends into the proximal transverse aortic arch. b A 23-day-old boy on VA ECMO also for congenital heart disease with an intraluminal dissection flap of the ascending aorta (arrow). In this case, contrast was injected via a central venous catheter with the tip in the right atrium (note that the aorta is opacified). Both findings were suspected on echocardiography and confirmed on CT angiography

\section{Conclusion}

ECMO is a life-saving treatment in patients with respiratory or cardiac failure. The radiologic assessment of this device is very important. Having a basic understanding of the different ECMO modes, the various ECMO indications, the complex medical management of ECMO patients, and the wide range of serious ECMO complications allows the radiologist to be a more effective consultant. Since cannulae can be assessed by radiograph, CT and US, radiologists should be familiar with the details of ECMO cannulae. This includes a working knowledge of the various cannula types, the differing primary and accessory cannula configurations, the desired location of cannula drainage/reinfusion ports, and the patient's cardiovascular anatomy. It is each radiologist's responsibility to understand ECMO from both clinical and imaging perspectives. This will help detect and prevent complications.

\section{Compliance with ethical standards}

Conflicts of interest None

\section{References}

1. Hill JD, O'Brien TG, Murray JJ et al (1972) Prolonged extracorporeal oxygenation for acute post-traumatic respiratory failure (shocklung syndrome). Use of the Bramson membrane lung. $\mathrm{N}$ Engl $\mathrm{J}$ Med 286:629-634
2. Extracorporeal life support registry report. Available online: https:// www.elso.org/Registry/Statistics/InternationalSummary.aspx. Accessed 6 April 2018

3. Pavlushkov E, Berman M, Valchanov K (2017) Cannulation techniques for extracorporeal life support. Ann Transl Med 5:70

4. Makdisi G, Wang IW (2015) Extra corporeal membrane oxygenation $(E C M O)$ review of a lifesaving technology. J Thorac Dis 7:E166-E176

5. Extracorporeal Life Support Organization (ELSO) General Guidelines for All ECLS Cases; version 1.3, November 2013. Available online: www.elso.org/Portals/0/IGD/Archive/FileManager/ 929122ae88cusersshyerdocumentselsoguidelinesgeneralalleclsversion1. 3.pdf. Accessed 6 April 2018

6. Wildschut ED, Ahsman MJ, Allegaert K et al (2010) Determinants of drug absorption in different ECMO circuits. Intensive Care Med 36:2109-2116

7. Marasco SF, Lukas G, McDonald M et al (2008) Review of ECMO (extra corporeal membrane oxygenation) support in critically ill adult patients. Heart Lung Circ 17:S41-S47

8. Schmidt M, Pellegrino V, Combes A et al (2014) Mechanical ventilation during extracorporeal membrane oxygenation. Crit Care 18:203

9. Napp LC, Kühn C, Hoeper MM et al (2016) Cannulation strategies for percutaneous extracorporeal membrane oxygenation in adults. Clin Res Cardiol 105:283-296

10. Extracorporeal Life Support Organization (ELSO) Guidelines for Cardiac Failure; version 1.3, December 2013. Available online: www.elso.org/Portals/0/IGD/Archive/FileManager/ 518a079853cusersshyerdocumentselsoguidelinesforpediatriccardiacfailure1. 3.pdf. Accessed 6 April 2018

11. Kanji HD, Schulze CJ, Oreopoulos A et al (2010) Peripheral versus central cannulation for extracorporeal membrane oxygenation: a comparison of limb ischemia and transfusion requirements. Thorac Cardiovasc Surg 58: $459-462$ 
12. Mcllwain RB, Timpa JG, Kurundkar AR et al (2010) Plasma concentrations of inflammatory cytokines rise rapidly during ECMOrelated SIRS due to the release of preformed stores in the intestine. Lab Investig 90:128-139

13. Aiyagari RM, Rocchini AP, Remenapp RT, Graziano JN (2006) Decompression of the left atrium during extracorporeal membrane oxygenation using a transseptal cannula incorporated into the circuit. Crit Care Med 34:2603-2606

14. Lambert L, Grus T, Balik M et al (2017) Hemodynamic changes in patients with extracorporeal membrane oxygenation (ECMO) demonstrated by contrast-enhanced CT examinations - implications for image acquisition technique. Perfusion 32:220-225

15. Short BL (2005) The effect of extracorporeal life support on the brain: a focus on ECMO. Semin Perinatol 29:45-50

16. Extracorporeal Life Support Organization (ELSO) Guidelines for Neonatal Respiratory Failure; version 1.3 December 2013. Available online: https://www.elso.org/Resources/Guidelines.aspx. Accessed 1 Sept 2017 